\section{Uso e abuso de benzodiazepínicos na atenção primária à saúde: práticas profissionais no Brasil e em Cuba}

\author{
Use and abuse of benzodiazepines in primary \\ healthcare: professional practices in \\ Brazil and Cuba
}

\section{Uso y abuso de benzodiacepinas en la atención primaria de salud: prácticas profesionales en Brasil y Cuba}

Claudia Fegadolli 1

Niurka Maria Dupotey Varela 2

Elisaldo Luis de Araújo Carlini 1

doi: 10.1590/0102-311X00097718

\section{Resumo}

O presente estudo buscou compreender aspectos assistenciais presentes na base da utilização indiscriminada de benzodiazepínicos. Com base numa parceria entre uma universidade brasileira e uma cubana, buscou-se o entendimento das práticas relacionadas ao uso desses medicamentos na atenção primária $e$ dos sentidos que profissionais de saúde atribuem a elas. A investigação integrou um estudo de casos múltiplos realizado nos municípios de São Paulo e Diadema (Brasil) e Santiago de Cuba (Cuba). O trabalho de campo adotou como estratégias de coleta de dados entrevistas individuais e grupos focais. Os dados, analisados tematicamente, revelaram cinco temas: (i) terra de ninguém: a ausência de gestão sobre o uso dos benzodiazepínicos pelos profissionais da atenção básica; (ii) indicação inadequada: o benzodiazepínico prescrito psra situações injustificáveis; (iii) salvação e perdição: o medicamento como atenuante da dificuldade de atuação na saúde mental pelos profissionais da atenção primária; (iv) pouco empoderamento dos profissionais da atenção primária para atuação na saúde mental; $e(v)$ cuidado fragmentado: a desarticulação da rede de atenção psicossocial. A pouca apropriação das questões da saúde mental pelos profissionais da atenção primária, a fragmentação do cuidado, a sobrecarga de trabalho com temas considerados prioritários, as deficiências na disponibilidade de recursos terapêuticos e o pouco investimento em formação específica contribuem para o uso não adequado de benzodiazepinicos. Independentemente dos contextos sanitários estudados, os desafios são semelhantes para os sistemas de saúde e só podem ser enfrentados se convertidos em prioridade para a gestão das organizações e para o conjunto dos profissionais.

Ansiolíticos; Serviços Farmacêuticos; Saúde Mental; Sistemas de Saúde; Atenção Primária à Saúde

\author{
Correspondência \\ C. Fegadolli \\ Alameda Itu 282, apto. 72, São Paulo, SP 01421-000, Brasil. \\ cfegadolli@hotmail.com \\ 1 Universidade Federal de São Paulo, São Paulo, Brasil. \\ 2 Universidad de Oriente, Santiago de Cuba, Cuba.
}




\section{Introdução}

Há décadas se reconhece o uso indiscriminado de benzodiazepínicos no mundo, principalmente a utilização por longos períodos e em situações injustificadas 1,2,3,4,5,6. Estão entre os cinco medicamentos controlados mais vendidos no Brasil, com maior consumo nas regiões com alta densidade populacional e maior número de médicos 7 . No Município de São Paulo, correspondem ao tipo de medicamento psicotrópico mais utilizado, à frente de antidepressivos e antipsicóticos 8 . O uso disseminado de benzodiazepínicos é um problema de saúde pública também nos países da América Latina e Caribe, como Chile, Venezuela, Uruguai e Argentina 9. Em Cuba, embora sejam escassas as estatísticas sobre o consumo, já se identificou o uso problemático, especialmente entre idosos 10,11,12.

A ampla prescrição e uso de benzodiazepínicos são resultados de práticas que correspondem ao processo de medicalização da sociedade, em que se consideram problemas médicos tratáveis diversas situações consideradas como desvios de normalidade nos processos naturais da vida ou de normas sociais 13 . Nesse contexto, são exemplos o nervosismo do cotidiano, a necessidade de mascarar as dificuldades da vida ou, ainda, de lidar com o envelhecimento 14,15 .

Os elevados perfis de utilização trazem importantes consequências, que vão além das reações adversas, efeitos colaterais e paradoxais que reconhecidamente os benzodiazepínicos produzem quando usados nas situações e limites preconizados. Há, também, impactos do uso prolongado desses medicamentos, como déficits cognitivos, alterações motoras, sedação excessiva, tolerância e dependência, entre outros efeitos decorrentes da utilização inapropriada ou abusiva 16,17,18. Porém, mesmo com os alertas sobre a segurança, o consumo dos benzodiazepínicos tem crescido no mundo todo, exceto quando se implementam medidas específicas, principalmente as restritivas de financiamento nos serviços de saúde 19,20. Nos Estados Unidos, por exemplo, o número de adultos que recebeu ao menos uma prescrição de benzodiazepínicos passou de 8,1 para 13,5 milhões no período de 1996 a 2016, com consequências gravíssimas como overdose e morte 21.

No Brasil, a maior parte das prescrições de benzodiazepínicos é emitida em serviços de atenção primária, em que os médicos relatam ter pouco tempo para consultas e para o desenvolvimento de estratégias terapêuticas alternativas no tratamento da insônia e ansiedade, que são os principais motivos do consumo 22,23,24. Entre outros possíveis fatores, o uso fora das recomendações pelas autoridades sanitárias é impulsionado por problemas na qualidade da assistência à saúde e, assim como a assistência impacta o uso, este eleva os custos do cuidado e gera novas demandas 25 .

Embora o problema seja bastante reconhecido, pouco se avança em medidas efetivas de melhoria nos padrões de consumo, o que gera a necessidade de se analisarem em profundidade os aspectos assistenciais que estão na base da utilização indiscriminada dos benzodiazepínicos. Assim, valendose da parceria entre uma universidade brasileira e uma cubana, esta pesquisa buscou o entendimento sobre as práticas sanitárias na provisão de benzodiazepínicos na atenção primária e acerca dos sentidos que profissionais de saúde atribuem a elas.

\section{Método}

A pesquisa integrou um estudo de casos múltiplos $26 \mathrm{em}$ serviços de atenção primária à saúde, a partir de parceria entre a Universidade Federal de São Paulo e a Universidade do Oriente de Santiago de Cuba. Nos municípios brasileiros de São Paulo e Diadema, Estado de São Paulo, o trabalho foi realizado em seis unidades básicas de saúde, das quais cinco operam com Estratégia Saúde da Família (ESF). Em Cuba, foi desenvolvido no Município de Santiago de Cuba, em uma região atendida por uma farmácia central municipal e 14 policlínicos com modelo de médico de família. A seleção dos serviços de saúde foi intencional, de acordo com a localização das unidades universitárias nos municípios. Foram realizadas entrevistas individuais em profundidade, grupos focais e observação participante 27 dos atendimentos médico e farmacêutico, além do momento de espera dos usuários para o atendimento com estes dois profissionais. O trabalho de campo foi conduzido, no Brasil, nos períodos de dezembro de 2016 a abril de 2017, em São Paulo e, de setembro a novembro de 2017, em Diadema. Em Cuba, compreendeu o período de maio a agosto de 2017. A mesma equipe de investigação atuou nos 
dois países, sendo uma pesquisadora principal brasileira e uma assistente cubana. A diversidade das técnicas de coleta de dados adotadas contribuiu com a ampla compreensão dos pesquisadores acerca das relações e especificidades existentes nos cenários estudados, embora, neste artigo, os resultados apresentados se refiram às entrevistas e grupos focais, cujos conteúdos foram gravados, transcritos na íntegra e analisados tematicamente 28.

Foram realizadas 22 entrevistas individuais e seis grupos focais, além de 95 horas de observação, determinadas pela saturação dos dados. No Brasil foram entrevistados individualmente cinco farmacêuticos, dois médicos psiquiatras, três psicólogos e três gerentes de serviço. Doze médicos generalistas, um ginecologista e seis enfermeiros participaram de grupos focais, separados por categoria profissional, médico e enfermeiro. Em Cuba, todas as entrevistas foram individuais com um farmacêutico, duas gestoras e seis médicos de família. A condução das entrevistas e grupos focais foi apoiada em um roteiro semiestruturado que convidava os participantes a falarem livremente sobre o que conheciam a respeito do uso de benzodiazepínicos pela população atendida em suas unidades de trabalho, sobre possíveis dificuldades que tinham com o tema e como visualizavam possibilidades de melhoria no uso destes medicamentos pela população.

Embora no planejamento da pesquisa estivesse prevista a apresentação dos resultados de maneira comparativa entre os dois países, os achados foram apresentados conjuntamente devido à convergência dos discursos e práticas. Os cinco temas revelados foram nomeados ilustrativamente segundo as categorias analíticas dos pesquisadores que, às vezes, coincidiram com fragmentos de discursos dos participantes. São assim apresentados: "terra de ninguém: a ausência de gestão sobre o uso dos benzodiazepínicos pelos profissionais da atenção básica”; "indicação inadequada: o benzodiazepínico prescrito para situações injustificáveis"; "salvação e perdição: o medicamento como atenuante da dificuldade de atuação na saúde mental pelos profissionais da atenção primária"; "pouco empoderamento dos profissionais da atenção primária para atuação na saúde mental” e, por fim, "cuidado fragmentado: a desarticulação da rede de atenção psicossocial".

O projeto foi aprovado pelo Comitê de Ética em Pesquisa da Universidade Federal de São Paulo (número 1.071.384) e pelo Comitê de Ética do Município de São Paulo (CAAE 43.230.815). Em Cuba, foi autorizado pela Direção de Farmácia e Óptica de Santiago de Cuba e pela Universidade do Oriente.

\section{Resultados e discussão}

A convergência dos dados dos dois países, que possibilitou sua apresentação de maneira integrada, ocorre pelas semelhanças identificadas entre os sistemas de saúde. O sistema nacional de saúde brasileiro busca inspirações no sistema cubano, do ponto de vista da oferta de saúde gratuita, integral, participativa e equânime para a totalidade da população. Além disso, embora no Brasil coexistam variações no modelo de atenção básica à saúde, prevalece a ESF, modelo semelhante ao que assiste os cubanos, focado na atuação do médico e enfermeiro de família.

Há, no entanto, diferenças importantes. Em Cuba, quase a totalidade dos investimentos em saúde é custeada pelo Estado, e no Brasil mais da metade dos recursos são de origem privada, embora compensados por mecanismos de isenção fiscal 29. O privado também está presente na oferta de medicamentos no Brasil, onde, muitas vezes, gestores e prescritores são estimulados a prescrever por meio das estratégias de mercado da indústria farmacêutica, o que não ocorre em Cuba, onde $100 \%$ da disponibilidade de medicamentos são de provimento estatal e onde não existe propaganda dirigida de produtos farmacêuticos, principalmente daqueles sob controle especial, como ocorre no Brasil.

O Sistema Único de Saúde (SUS) brasileiro atende, na atenção básica, predominantemente indivíduos de renda baixa e que não podem arcar com planos privados de saúde, o que não se aplica aos procedimentos de alta complexidade e de componentes especializados, altamente demandados por todos os extratos sociais. A questão da classe social é a principal diferença. No Brasil existe a classificação socioeconômica da sociedade em oito extratos (A1, A2, B1, B2, C1, C2, D e E), de acordo com renda, moradia e consumo 30, em Cuba, segundo sua Constituição Federal, não há diversidade de classe social. Qualquer cidadão, de acordo com méritos e capacidades, tem acesso a todos os postos de trabalho do Estado, da administração pública e da produção e prestação de serviços, além de ascensão a todas 
as hierarquias das forças armadas e da ordem de segurança e interna. No entanto, transformações recentes têm gerado uma reconformação classista complexa, com alguns padrões de desigualdade associados a rendimentos e reformas do emprego 31.

O desenvolvimento do estudo, que teve duração de três meses de vivência em Cuba, evidenciou que há diferenças quanto ao poder de compra entre as famílias, porém não tão gritantes como no Brasil. Com a crescente abertura que vive hoje o sistema socialista, é permitido que os cidadãos tenham pequenos negócios, predominantemente na área de transporte e turismo. Essa possibilidade gera renda extra e o acesso ao consumo de bens e serviços anteriormente mais limitados aos cubanos, como roupas, restaurantes, lazer e viagens. Todos vivenciam, no entanto, os mesmos problemas estruturais, como aqueles relacionados ao transporte e disponibilidade de produtos e alimentos. Porém, não se observa em Cuba o que acontece no Brasil, onde há pessoas que moram nas ruas e não têm alimento suficiente para viver. Mesmo com o consumo restrito, todos têm, naquele país, o acesso aos direitos mínimos condizentes com a dignidade humana, como alimentos, saúde, educação, segurança e moradia, ainda que existam precariedades, conforme observamos nas cidades visitadas. Esclarecidas as principais semelhanças e diferenças nos contextos de análise, segue a apresentação e discussão dos temas identificados:

\section{Terra de ninguém: a ausência de gestão sobre o uso dos benzodiazepínicos pelos profissionais da atenção básica}

Este tema é central na análise, uma vez que representa o cenário de práticas em que os profissionais da atenção primária agem independentes uns dos outros e alienadamente às recomendações para que benzodiazepínicos não sejam empregados por período superior a quatro semanas 32 . Os profissionais reconhecem o uso indiscriminado de benzodiazepínicos no cotidiano, porém, parece não identificar suas próprias condutas como determinantes deste cenário. Consideram que apenas mudanças na regulação, nas diretrizes da gestão ou no comportamento de outros profissionais solucionariam o problema, mesmo que hipoteticamente.

"Eu penso que a única maneira de enfrentar seria que não nos fosse permitido emitir as receitas e que só pudesse fazer isso um psiquiatra, mas tem poucos psiquiatras para a quantidade de pacientes" (Médico de família - Cuba, entrevista individual).

O controle sanitário vigente parece não ser suficiente para racionalizar o uso, pensamento que coincide com Weaver 19 e Lembke et al. 33, que defendem que as regras de prescrição de benzodiazepínicos deveriam ser tão rigorosas quanto as de opiáceos.

A pressão dos usuários é uma realidade cotidiana que dificulta o posicionamento do prescritor.

"As pessoas são muito autônomas com seus tratamentos, mas você não vê um diabético tão autônomo, eu as vejo autônomas com esse problema, insônia e ansiedade" (Farmacêutica - Cuba, entrevista individual).

"Te assediam e pressionam: e por que você não quer me dar? Antes eu vinha e você me dava, e por que você tem que me perguntar tanto para me dar?" (Médico de família - Cuba, entrevista individual).

A pressão exercida pelos usuários influencia a conduta dos prescritores, assim como já verificaram Rosa et al. 34, cujo estudo constatou a emissão de prescrições por médicos brasileiros em atendimento à solicitação de usuários que já consomem psicofármacos, sem avaliação da necessidade da indicação, o que também foi identificado em um estudo na Noruega 35. Nas práticas em saúde, a interação entre médico e paciente é um dos determinantes do nível de medicalização de uma sociedade e, neste caso, é extremamente relevante o fato de os usuários de benzodiazepínicos se apropriarem de seu problema de saúde e da forma de sua resolução atuando, consequentemente, no convencimento dos médicos para que prescrevam 36 .

Oferecer medicamentos em resposta a pressões pode indicar pouco empoderamento dos profissionais, decorrente de falta de acesso a processos de aprendizagem e de métodos de trabalho que permitam a tomada de decisões, além de dificuldades de reconhecimento de iniciativas com compartilhamento de responsabilidades. Esses comportamentos geram círculos viciosos:

"Não sabemos como resolver e somos também participantes porque nós é que estamos emitindo a receita que as pessoas usam" (Médico de família - Cuba, entrevista individual).

Sem estratégias claras para o enfrentamento, o uso irracional de benzodiazepínicos é produzido sem visibilidade. Para os sistemas de saúde, é como se fosse algo menos importante. 
"Eu nunca me atentei a acompanhar essa questão (...). Eu percebo que a maioria das prescrições é pra casos bem críticos, mas eu não tenho controle de todos" (Farmacêutico - Brasil, entrevista individual).

"Tenho focado em outros grupos de risco, trabalhado com cardiopatias, diabetes, e como sou uma pessoa só não posso dar conta de tudo" (Farmacêutico - Cuba, entrevista individual).

"O problema está no tempo, temos nosso tempo em função do mosquito, dengue, Zika, chikungunha, diarreia (...). Estamos com muito trabalho pelo aspecto epidemiológico" (Médico de família - Cuba, entrevista individual).

Os profissionais parecem esperar que uma ação externa dispare atitudes no cotidiano e atribuem os problemas ao contexto.

"Temos que ter um suporte teórico, estudos e, no país todo, eu só vi duas investigações, então é terra de ninguém, temos que convertê-la em nossa terra" (Farmacêutico - Cuba, entrevista individual).

Ao não serem adotadas abordagens de investigação ou educativas que norteiem as práticas diárias, as impressões seguem sendo superficiais, diluídas entre tantas demandas cotidianas. Como a questão não é visualizada, os profissionais não se apropriam dela e veem o uso crônico com naturalidade.

"Tem usuários aqui que já fazem uso de benzodiazepínicos há mais de 15 anos, tem uns que já fazem uso há quase 20 anos" (Farmacêutico - Brasil, entrevista individual).

A naturalização do uso por períodos prolongados aparece repetidamente nos discursos e pode ser exemplificada pela fala de uma farmacêutica.

"Há um controle logístico, sabe-se quem está comprando, são exigidos determinados elementos, a prescrição é restritiva, uma só pessoa não pode levar duas ou três receitas, isso é um controle, porém para a aquisição. Mas, uma vez que essa pessoa levou os medicamentos, ai é onde estamos falhando, o que acontece com esse paciente?" (Farmacêutico - Cuba, entrevista individual).

Porém, mesmo que o sistema não identifique o problema, que segue quase invisível, ele é percebido pelos profissionais individualmente.

\section{Indicação inadequada: o benzodiazepínico prescrito para situações injustificáveis}

Os profissionais da atenção primária referem que dificilmente iniciam novos tratamentos, mas que dão continuidade a prescrições anteriores, a maior parte com indicação inadequada, conforme sintetiza uma das falas:

"A principal causa que leva ao uso é um quadro depressivo que traz insônia. É um diagnóstico de depressão mal feito, já entram direto no benzodiazepínico. Eles vão tratar a insônia, a paciente continua depressiva e cada vez mais aumentam a dose do benzodiazepínico. Outra causa é a menopausa, a gente sabe que está associada com quadro depressivo, de insônia, de alteração do humor, a maioria dos clínicos associa isso a um quadro de depressão, mas eles não tratam a menopausa, eles tratam a insônia ou a depressão. E em terceiro lugar eu pego os casos que têm problema familiar, que são os que eu tenho mais dificuldade de tirar" (Médico ginecologista - Brasil, grupo focal).

A prática de indicar benzodiazepínicos na fase do climatério é reforçada.

"Você tem calor, aí te dá frio, você se sente irritada, às vezes não quer ninguém perto de você (...) são coisas normais porque são hormônios caindo no organismo, mas que te fazem mal, você se sente desesperada (...) por isso parece que mulheres usam mais, por causa da menopausa, e ai vem a etapa da terceira idade" (Médico de família - Cuba, entrevista individual).

Vir a etapa da terceira idade significa que a usuária entra num ciclo do qual dificilmente consegue sair.

"Por serem pacientes que usam há tanto tempo é difícil tirar. Chega um momento que você diz 'você já tá velhinha' ou 'você tem uma idade que já não deve mais tomar isso', mas eles se sentem bem com o medicamento porque têm dependência, se eles não têm o medicamento, não estão bem” (Médico de família - Cuba, entrevista individual).

A falta de recursos farmacológicos, como antidepressivos, e a necessidade de atender às demandas dos pacientes são determinantes. Além disso, as imprecisões diagnósticas e terapêuticas ampliam o número de usuários, o que é confirmado na literatura sobre o tema 22,23,37.

Há, entretanto, olhares que captam outra dimensão no processo que gera o uso dos medicamentos.

"O paciente não vem com essa referência de que existe uma medicação, ele não vem só com isso, ele vem 
pedindo uma ajuda. Quem, muitas vezes, oferece a ajuda medicamentosa é o profissional, ao invés de ter um olhar do que está produzindo a insônia" (Psicólogo - Brasil, entrevista individual).

\section{Salvação e perdição: o medicamento como atenuante da dificuldade de atuação na saúde mental pelos profissionais da atenção primária}

Definido como solução possível, o benzodiazepínico rapidamente assume o status de mal necessário. No discurso e nas práticas observadas, a prescrição continuada é avaliada como uma necessidade do paciente e ocorre mesmo com a compreensão de que o uso prolongado acarreta riscos. Os profissionais acreditam que, uma vez iniciado o uso, não se pode parar.

"Fazem da medicação um salvador da pátria para tentar mascarar um pouco a vida que é tão difícil (...). O medicamento é a forma de escape, de ficar melhor e conseguir dar conta da vida" (Farmacêutico - Brasil, entrevista individual).

"Nunca nenhum caso que usava durante um bom tempo descontinuou, eu nunca vi em cinco anos" (Farmacêutico - Brasil, entrevista individual).

Uma vez introduzido o benzodiazepínicos, a suposta solução é convertida em problema, com a percepção de que a dependência assume o controle, difícil de ser contornada por profissionais e pelos próprios usuários.

"Eles não se preocupam que não haja outras coisas. Não há nitrazepam, não há diazepam, não há clorodizepóxido nas farmácias e as pessoas criam um alarme enorme. Pode não haver metformina, não haver enalapril, não haver atenolol, isso não lhes preocupa" (Médico de família - Cuba, entrevista individual).

"Porque é algo como o fumante: sabe que o cigarro vai causar danos, mas continua fumando; o bebedor, sabe que o álcool vai matá-lo, mas continua bebendo. É igualzinho, acontece o mesmo com os comprimidos" (Médico de família - Brasil, grupo focal).

A impressão dos profissionais é respaldada por autores que estudaram a experiência dos usuários com os benzodiazepínicos e identificaram que ocorre, ao mesmo tempo, situação de alívio e de sofrimento com a dependência 38,39. O desconforto dos profissionais, entretanto, não impede que os medicamentos continuem sendo prescritos por período prolongado, também por sua aparente segurança.

"Destrói, mas a longo prazo, pelos acidentes que podem apresentar, porque se torna viciado no medicamento, mas na verdade um acidente grave não se vê” (Médico de família - Cuba, entrevista individual).

Mesmo com a ideia latente de que há problemas relativos à segurança, a possível dificuldade em se atribuir diretamente aos benzodiazepínicos os riscos associados ao seu uso prolongado torna-os, de certa maneira, invisíveis. Os efeitos adversos podem ser confundidos com outras causas que levam a eventos como quedas, perda de memória, desenvolvimento de demência e acidentes de trânsito 40. Para os prescritores, os pacientes desconhecem ou negam os conhecidos riscos e, eles próprios, tendem a crer que os usuários os podem tolerar bem. O que ocorre, consequentemente, é que a dificuldade de estabelecer relações diretas de causa e efeito diminui a percepção sobre os riscos dos benzodiazepínicos e, assim, a chance de que o usuário tenha a intenção de descontinuar o uso. No mesmo sentido, a não visualização de tais efeitos pelo prescritor também inibe o enfrentamento do problema, o que exige que a construção de novas percepções seja considerada no delineamento de estratégias educativas e de racionalização do uso de benzodiazepínicos 41,42,43.

Mas, ainda que pouco visualizados de maneira concreta, a percepção dos riscos está sempre presente nos discursos dos profissionais estudados, que mostram uma grande insatisfação por se verem induzidos às prescrições sistemáticas, seja pelas pressões dos usuários, seja pelas condições de trabalho vivenciadas.

\section{Pouco empoderamento dos profissionais da atenção primária para atuação na saúde mental}

Perante as pressões dos usuários, a falta de formação para atuar em saúde mental, o pouco tempo para escuta e avaliação nas consultas ou outras insuficiências nas condições de trabalho, os profissionais sentem-se impotentes:

"As pessoas pensam que a atenção básica não quer fazer nada. Não é isso, a gente quer fazer um trabalho com respaldo da especialidade, não ficar só trocando, trocando, trocando [receitas]" (Médico de família - Brasil, grupo focal). 
Os profissionais percebem a atenção primária como um ponto de renovação de receitas.

"[A maioria das receitas] são renovação, às vezes, pode-se passar um sedantezinho para que se resolva uma crise por um tempo, agora se tem uma coisa mais prolongada é porque tem um seguimento com o psiquiatra" (Médico de família - Cuba, entrevista individual).

A influência das condições de trabalho é identificada.

"O benzodiazepinico é o que tem na mão, você vai fazer o quê? Se você não tem condição de dar suporte pra todo esse pessoal que vem pra consulta e cada vez vem mais, não tem jeito, você tem que fazer alguma coisa" (Médico de família - Brasil, grupo focal).

Em Cuba, embora haja disponibilidade de terapias de apoio, elas não parecem ser priorizadas, estão disponíveis, mas faltam políticas de implantação.

"Contamos, por exemplo, com medicinas naturais, tradicionais, homeopatia, fitofármacos e tudo o que se pode utilizar, mas as pessoas não interiorizaram, ainda, que isso é bom (...). Sabem, mas é como se o comprimido fosse melhor (...). O problema é o costume, quanto mais se socializa a cultura urbana, menos se quer utilizar o que os ancestrais utilizavam (Médico de família - Cuba, entrevista individual).

Quanto às questões de formação, as inseguranças no campo da saúde mental são identificadas principalmente entre os médicos de família.

"Fazer grupo de nutrição é uma coisa, fazer grupo de dependência de benzo você precisa de suporte psiquiátrico, não é muito fácil" (Médico de família - Brasil, grupo focal).

O cuidado em saúde mental, além dos aspectos clínicos, demanda do profissional recursos afetivos, posturas profissionais voltadas à escuta e competências que, às vezes, não são proporcionados pelo ensino formal, que falha no desenvolvimento de habilidades importantes para este tipo de atuação $44,45,46$.

\section{Cuidado fragmentado: a desarticulação da rede de atenção psicossocial}

Embora exista alguma interação da atenção primária com a especialidade psiquiatria, que ocorre efetivamente na discussão de casos ou apoio matricial, a fragmentação do cuidado é uma realidade. No Brasil, onde a atenção primária conta com o apoio matricial em saúde mental como estratégia de apoio técnico e de educação permanente 47,48 , este parece não ser suficiente para sustentar o uso racional e seguro de benzodiazepínicos.

O compartilhamento e a interprofissionalidade são bastante restritos. Os problemas de insônia, ansiedade e outros de saúde mental aparentam estar à parte no processo de cuidado na atenção primária à saúde e o tema parece ser assunto para o psiquiatra. O que ocorre nos dois países é que psiquiatras, médicos de unidades de pronto-atendimento e de outros serviços prescrevem o benzodiazepínicos, o médico de família renova a receita com o apoio do enfermeiro e o usuário retira o medicamento na farmácia, onde o farmacêutico apenas controla a quantidade retirada. Porém, a atenção primária não faz o acompanhamento do uso e dos efeitos da utilização dos benzodiazepínicos e o médico de família não sente que pode interferir.

"Nós não podemos lidar muito com isso como não lidaríamos com o cardiologista que põe um tratamento $e$ você diz que não está bom. Já vem de escala 'ah!, mas isso me mandou o psiquiatra, isso me prescreveu o psiquiatra" (Médico de família - Cuba, entrevista individual).

As consultas com especialistas não são vistas como solução.

"Não adianta reencaminhar pro psiquiatra que ele vai voltar com outra receita de benzo [benzodiazepínico]" (Médico de família - Brasil, grupo focal).

Outro elemento corraborador é a facilidade de obtenção de receituário em diversos pontos de atenção à saúde, sem controle da informação.

"Pode ser que ele não consiga comigo e consiga com outro" (Médico de família - Cuba, entrevista individual).

A desarticulação ocorre também do ponto de vista do trabalho farmacêutico. Em nenhuma das farmácias observou-se dispensação com orientação nem interlocução com médicos sobre a necessidade de reavaliação do tratamento.

"É que, por exemplo, uma vez prescrito eu fico com receio de dar uma abordagem" (Farmacêutico - Brasil, entrevista individual). 
A fragmentação do trabalho é conhecida limitadora do papel da atenção primária na gestão do cuidado, com problemas relacionados a falhas na comunicação e colaboração interprofissional 49. Essa realidade se aplica aos cuidados em saúde mental, que estão entre as muitas responsabilidades da atenção básica, para os quais, embora seja amplo o aporte teórico e diretrizes de gestão, ainda há carência de maior integração, tanto por deficiências na formação dos profissionais, como na estruturação da rede, incluindo recursos terapêuticos.

Para a atenção primária à saúde parece ser um grande desafio ter de garantir ação resolutiva para os problemas considerados primários e, ao mesmo tempo, dar conta de especificidades como a saúde mental. Em Cuba, que resolve de maneira bastante efetiva as demandas na atenção primária à saúde, exige-se dos profissionais e da gestão constante esforço para lidar com a falta de insumos e produtos para a saúde pelos escassos recursos econômicos. No Brasil, além desses mesmos aspectos, dificultam ainda mais os cuidados de saúde mental na atenção primária à saúde os problemas de acolhimento e de formação e consolidação de vínculos entre os profissionais e os usuários, além de deficiências no sistema de referência e contrarreferência 50,51 .

Na complexidade desse cenário, embora os profissionais reconheçam que mudanças são possíveis na somatória de esforços, as ações são mencionadas como possibilidade quase remota, pouco ou nada concreta.

\section{Considerações finais}

Ainda que sejam coerentes com outras publicações, por se tratar de estudo de caso, as generalizações dos resultados desta pesquisa devem considerar a realidade da população e dos sistemas de saúde estudados.

Depreende-se do estudo que a pouca apropriação das questões da saúde mental pelos profissionais da atenção primária, a fragmentação do cuidado, a sobrecarga com outros temas considerados prioritários, as deficiências na disponibilidade de recursos terapêuticos e o pouco investimento em formação específica contribuem para o uso não adequado de benzodiazepínicos nos serviços pesquisados.

Nesse cenário, favorece-se a geração de usuários de benzodiazepínicos que buscam o alívio de sintomas que poderiam ser tratados de outra maneira e pouco ou nenhum controle efetivo sobre o uso. Essa realidade, porém, somente pode ser transformada se for convertida em prioridade para a gestão das organizações e para o conjunto dos profissionais. São necessários, sobretudo, investimentos em mecanismos de empoderamento dos profissionais, como educação continuada ou permanente, reorganização do trabalho e dos serviços, favorecendo a interprofissionalidade e articulação, além de políticas de implementação de recursos terapêuticos alternativos.

\section{Colaboradores}

C. Fegadolli planejou o estudo, executou desde a coleta de dados até a discussão dos resultados e redigiu o artigo. N. M. D. Varela contribuiu com o planejamento, coleta de dados e revisão do artigo. E. L. A. Carlini orientou a pesquisa e revisou o artigo.

\section{Informações adicionais}

ORCID: Claudia Fegadolli (0000-0002-2991-0206); Niurka Maria Dupotey Varela (0000-0003-21101743); Elisaldo Luis de Araújo Carlini (0000-00029181-6659).

\section{Agradecimentos}

À Coordenação de Aperfeiçoamento de Pessoal de Nível Superior (Capes), pelo apoio concedido no contexto do projeto 193/14 do Programa CapesMES Cuba Projetos - Edital 46/2013. 


\section{Referências}

1. Donoghue J, Lader M. Usage of benzodiazepines: a review. Int J Psychiatry Clin Pract 2010; 14:78-87.

2. Dell'osso B, Albert U, Atti AR, Carmassi C, Carrà G, Cosci F, et al. Bridging the gap between education and appropriate use of benzodiazepines in psychiatric clinical practice. Neuropsychiatr Dis Treat 2015; 11:1885-909.

3. Lader M. Benzodiazepines revisited - will we ever learn? Addiction 2011; 106:2086-109.

4. Kapczinski F, Amaral OB, Madruga M, Quevedo J, Busnello JV, Lima MS. Use and misuse of benzodiazepines in Brazil: a review. Subst Use Misuse 2001; 36:1053-69.

5. Ruiz I, Offermanns J, Lanctôt KL, Busto U. Comparative study on benzodiazepine use in Canada and Chile. J Clin Pharmacol 1993; 33:124-9.

6. Sonnenberg CM, Bierman EJ, Deeg DJ, Comijs HC, van Tilburg W, Beekman AT. Ten-year trends in benzodiazepine use in the Dutch population. Soc Psychiatry Psychiatr Epidemiol 2009; 47:293-301.

7. Azevedo AJP, Araújo AA, Ferreira MAF. Consumo de ansiolíticos benzodiazepínicos: uma correlação entre dados do SNGPC e indicadores sociodemográficos nas capitais brasileiras. Ciênc Saúde Colet 2016; 21:83-90.

8. Blay SL, Fillenbaum GG, Pitta JC, Peluso ET. Factors associated with antidepressant, anxiolytic, and other psychotropic medication use to treat psychiatric symptoms in the city of São Paulo, Brazil. Int Clin Psychopharmacol 2014; 29:157-65

9. Sola V. 4 millones de cajas de benzodiazepinas se venden en Chile al año. Noticias Universidad San Sebastián 2015; 23 sep. http://www. uss.cl/newsletter-uss/2015/09/23/4-millo nes-de-cajas-de-benzodiacepinas-se-vendenen-chile-al-ano/.

10. Alfonso Hidalgo A, Prezaza González LM, Cabrera Arteaga D. Caracterización del consumo de benzodiazepinas en una farmacia del municipio de Santa Clara. Revista del Hospital Psiquiátrico de La Habana 2015; 12:1-5.

11. Oropeza Pupo DI, Calero González LM, Torrez Suárez R. Caracterización de la prescripción de benzodiazepinas en adultos mayores en un consultorio de la atención primara de salud. Correo Científico Médico de Holguín 2012; 16:1-13.

12. Sánchez LI, Hernández FF. Consumo de benzodiazepinas en pacientes geriátricos del consultorio \#12, policlínico "Campo Florido"'. Rev Cubana Farm 2010; 44:346-53.

13. Conrad P. The medicalization of society: on the transformation of human conditions into treatable disorders. Baltimore: Johns Hopkins University Press; 2007.

14. Pérodeau G, Grenon É, Grenier S, O’Connor K. Systemic model of chronic benzodiazepine use among mature adults. Aging Mental Health 2015; 20:380-90.
15. Mendonça RT, Carvalho ACD, Vieira EM, Adorno RCF. Medicalização de mulheres idosas e interação com consumo de calmantes. Saúde Soc 2008; 17:95-106.

16. Gage SB, Moride Y, Ducruet T, KurthT, Verdoux $\mathrm{H}$, Tournier $\mathrm{M}$, et al. Benzodiazepine use and risk of Alzheimer's disease: case-control study. BMJ 2014; 349:g5205.

17. Gray SL, Dublin S, Yu O, Walker R, Anderson M, Hubbard RA, et al. Benzodiazepine use and risk of incident dementia or cognitive decline: prospective population based study. BMJ 2016; 352:i90.

18. Johnson B, Streltzer J. Risks associated with long-term benzodiazepine use. Am Fam Physician 2013; 88:224-6.

19. Weaver MF. Prescription sedative misuse and abuse. Yale J Biol Med 2015; 88-247-56.

20. Hoebert JM. Reimbursement restriction and moderate decrease in benzodiazepine use in general practice. Ann Fam Med 2012; 10:42-9.

21. Benzodiazepine handbook: the prescription drug danger. Northpoint Recovery 2017; 19 aug. https://www.northpointrecovery.com/ blog/benzodiazepine-handbook-forgottenprescription-danger/.

22. Firmino KF, Abreu MHNG, Perini E, Magalhães SMS. Utilização de benzodiazepínicos no serviço municipal de saúde de Coronel Fabriciano, Minas Gerais. Ciênc Saúde Colet 2012; 17:157-66.

23. Ferrari CKB, Bruti LF, Oliveira CC, Moraes EV, Toledo OR, David FL. Falhas na prescrição e dispensação de medicamentos psicotrópicos: um problema de saúde pública. Rev Ciênc Farm Básica Apl 2013; 34:109-16.

24. Almeida LM, Coutinho ESF, Pepe VLE. Consumo de psicofármacos em uma região administrativa do Rio de Janeiro: a Ilha do Governador. Cad Saúde Pública 1994; 10:5-16.

25. Berger A, Edelsberg J, Treglia M, Alvir JM, Oster G. Change in healthcare utilization and costs following initiation of benzodiazepine therapy for long-term treatment of generalized anxiety disorder: a retrospective cohort study. BMC Psychiatry 2012; 23:177.

26. Yin RK. Estudo de caso: planejamento e métodos. 5a Ed. Porto Alegre: Bookman; 2015.

27. Minayo MCS. O desafio do conhecimento: pesquisa qualitativa em saúde. 9a Ed. São Paulo: Editora Hucitec; 2013.

28. Bardin L. Análise de conteúdo. Lisboa: Edições 70; 1916.

29. Ocké-Reis CO. Gasto privado em saúde no Brasil. Cad Saúde Pública 2015; 31:1351-3.

30. Kamakura W, Mazzon JA. Critérios de estratificação e comparação de classificadores socioeconômicos no Brasil. Rev Adm Empres 2016; 56:55-70.

31. Camacho EF, Farias AP. Clase social y territorio en Cuba: miradas a los procesos de desigualdad socioclasista y espacial en la periferia habanera. Cadernos do Ceam 2013; 13:83-114. 
32. World Health Organization. Pharmacological treatment of mental disorders in primary health care. Geneva: World Health Organization; 2009.

33. Lembke A, Papac J, Humphreys K. Our other prescription drug problem. N Engl J Med 2018; 378:693-5.

34. Rosa FS, Monteiro MTM, Fortunato JJ, Galato D. A prescrição de psicotrópicos e a reavaliação médica. J Bras Psiquiatr 2012; 61:52-3.

35. Dybwad TB, Kjølsrød L, Eskerud J, Laerum E. Why are some doctors high-prescribers of benzodiazepines and minor opiates? A qualitative study of GPs in Norway. Fam Pract 1997; 14:361-8.

36. Conrad P. Medicalization and social control. Ann Rev Sociol 1992; 18:209-32.

37. Sisurapanont M, Garner P, Critchley J, Wongpakaran N. Benzodiazepine prescribing behavior and atitudes: a survey among general practioners practicing in northern Thailand. BMC Fam Pract 2005; 6:27.

38. Alvarenga JM, Loyola Filho AI, Giacomin KC, Uchoa E, Firmo JOA. Uso de benzodiazepínicos entre idosos: o alívio de "jogar água no fogo”, não pensar e dormir. Rev Bras Geriatr Gerontol 2015; 18:249-58.

39. Liebrenz M, Gehring MT, Buadze A, Caflisch C. High-dose benzodiazepine dependence: a qualitative study of patients' perception on cessation and withdrawal. BMC Psychiatry 2015; 15:116.

40. Moore N, Pariente A, Bégaud B. Why are benzodiazepines not yet controlled substances? JAMA Psychiatry 2015; 72:110-1.

41. López HP. Percepción de riesgo asociada a la prescripción continuada de benzodiacepinas em salud mental y atención primaria. Adicciones 2014; 26:184-6.

42. Martin P, Tamblyn R, Ahmed S, Tannenbaum C. A drug education tool developed for older adults changes knowledge, beliefs and risk perceptions about inappropriate benzodiazepine prescriptions in the elderly. Patient Educ Couns 2013; 92:81-7.
43. Damestoy N, Collin J, Lalande R. Prescribing psychotropic medication for elderly patients: some physicians' perspectives. CMAJ 1999; 161:43-5.

44. Damous I, Erlich H. O ambulatório de saúde mental na rede de atenção psicossocial: reflexões sobre a clínica e a expansão das políticas de atenção primária. Physis (Rio J.) 2017; 27:911-32.

45. Rodrigues WO, Mourão LC, Almeida ACV, Oliveira GS. Os limites do ensino teórico-prático da saúde mental na formação do profissional de saúde. Rev Port Enferm Saúde Mental 2016; (Spe 4):107-14.

46. Oliveira TTSS, Leme FRG, Godoy KRG. O cuidado começa na escuta: profissionais de saúde mental e as vicissitudes da prática. Mental 2009; 7:119-38.

47. Organização Pan-Americana da Saúde; Organização Mundial da Saúde. A atenção à saúde coordenada pela APS: construindo as redes de atenção no SUS: contribuições para o debate. Brasília: Organização Pan-Americana da Saúde/Organização Mundial da Saúde; 2011.

48. Bonfim IG, Bastos ENE, Góis CWL, Tófoli LF. Apoio matricial em saúde mental na atenção primária à saúde: uma análise da produção científica e documental. Interface (Botucatu) 2013; 17:287-300.

49. Coordenação de Gestão da Atenção Básica, Coordenação Geral de Saúde Mental, Departamento de Atenção Básica, Departamento de Ações Programáticas Estratégicas, Secretaria de Atenção à Saúde, Ministério da Saúde. Saúde mental e atenção básica: o vínculo e o diálogo necessários. Brasília: Ministério da Saúde; 2003.

50. Wenceslau LD, Ortega F. Mental health within primary health care and global mental health: international perspectives and Brazilian context. Interface (Botucatu) 2015; 19:1121-32.

51. Frateschi MS, Cardoso CL. Saúde mental na atenção primária à saúde: avaliação sob a ótica dos usuários. Physis (Rio J.) 2014; 24:545-65. 


\begin{abstract}
The current study aimed to grasp the healthcare aspects present at the basis of the indiscriminate use of benzodiazepines. Based on a partnership between a Brazilian university and a Cuban university, the study aimed to understand the practices related to the use of these drugs in primary care and the meanings healthcare workers assign to them. The research was part of a multiple case study in the cities of São Paulo and Diadema (Brasil), and Santiago de Cuba (Cuba). The fieldwork data collection strategy was based on individual interviews and focus groups. Data were analyzed thematically and yielded five themes: (i) no man's land: lack of management of benzodiazepine use by primary care workers; (ii) inadequate indications: the benzodiazepine prescribed for unjustifiable situations; (iii) salvation and perdition: the medicine as attenuating the difficulty of acting in mental health by primary care professionals; (iv) limited empowerment to work in mental health; and (v) fragmented care: dissociation of the psychosocial care network. Limited grasp of mental health issues by primary care workers, fragmented care, work overload with what are considered other priorities, deficiencies in the availability of therapeutic resources, and limited investment in specific training contribute to the inadequate use of benzodiazepines. Independently of the health contexts, the challenges are similar for the health systems and can only be confronted if they become a priority for the organizations' management and the health workers as a whole.
\end{abstract}

Anti-anxiety Agents; Pharmaceutical Services; Mental Health; Health Systems; Primary Health Care

\section{Resumen}

El presente estudio tuvo como meta comprender aspectos asistenciales existentes en relación con la utilización indiscriminada de benzodiacepinas. A partir de la colaboración entre una universidad brasileña y una cubana, se procuró el entendimiento mutuo de las prácticas relacionadas con el uso de esos medicamentos en la atención primaria, así como el sentido que los profesionales de la salud les atribuyen. La investigación integró un estudio de casos múltiples, realizado en los municipios de Sao Paulo y Diadema (Brasil) y Santiago de Cuba (Cuba). El trabajo de campo adoptó como estrategias de recogida de datos entrevistas individuales $y$ grupos focales. Los datos, analizados temáticamente, revelaron cinco temas que detallamos a continuación. (i) tierra de nadie: la ausencia de gestión sobre el uso de las benzodiacepinas por parte de los profesionales de la atención básica; (ii) indicación inadecuada: las benzodiacepinas para situaciones injustificables; (iii) salvación y perdición: la medicación como atenuación de la dificultad de actuar en la salud mental por los profesionales de la atención primaria; (iv) poco empoderamiento para trabajar en el ámbito de la salud mental y (v) cuidado fragmentado: la desarticulación de la red de atención psicosocial. La escasa asunción de las cuestiones de salud mental por parte de los profesionales de atención primaria, la fragmentación del cuidado, la sobrecarga de trabajo con temas considerados prioritarios, las deficiencias en la disponibilidad de recursos terapéuticos y la poca inversión en formación específica contribuyen al uso no adecuado de benzodiacepinas. Independientemente de los contextos sanitarios estudiados, los desafíos son semejantes en todos los sistemas de salud y sólo pueden enfrentarse si se convierten en prioridad para quienes gestionan instituciones, así como para el conjunto de profesionales.

Ansiolíticos; Servicios Farmacéuticos; Salud

Mental; Sistemas de Salud; Atención

Primaria de Salud
Recebido em 18/Mai/2018

Versão final reapresentada em 29/Jan/2019

Aprovado em 06/Fev/2019 